\title{
Scalar elliptic equations with a singular drift
}

\author{
Misha Chernobai, Timofey Shilkin
}

October 6, 2022

\begin{abstract}
We investigate the weak solvability and properties of weak solutions to the Dirichlet problem for a scalar elliptic equation $-\Delta u+b^{(\alpha)} \cdot \nabla u=f$ in a bounded domain $\Omega \subset \mathbb{R}^{2}$ containing the origin, where $f \in W_{q}^{-1}(\Omega)$ with $q>2$ and $b^{(\alpha)}:=$ $b-\alpha \frac{x}{|x|^{2}}, b$ is a divergence-free vector field and $\alpha \in \mathbb{R}$ is a parameter.
\end{abstract}

\section{Introduction and Main Results}

Assume $\Omega \subset \mathbb{R}^{2}$ is a bounded simply connected domain with a $C^{1}$-smooth boundary $\partial \Omega$ and $0 \in \Omega$. We consider the following boundary value problem:

$$
\left\{\begin{array}{c}
-\Delta u+\left(b-\alpha \frac{x}{|x|^{2}}\right) \cdot \nabla u=-\operatorname{div} f \quad \text { in } \quad \Omega, \\
\left.u\right|_{\partial \Omega}=0 .
\end{array}\right.
$$

Here $u: \Omega \rightarrow \mathbb{R}, b: \Omega \rightarrow \mathbb{R}^{2}$ and $f: \Omega \rightarrow \mathbb{R}^{2}$ are given functions and $\alpha \in \mathbb{R}$ is a parameter. We always assume that the vector field $b$ satisfies the assumptions

$$
b \in L_{2, w}(\Omega), \quad \operatorname{div} b=0 \quad \text { in } \quad \mathcal{D}^{\prime}(\Omega),
$$

where $L_{2, w}(\Omega)$ is a weak Lebesgue space equipped with the norm

$$
\|b\|_{L_{2, w}(\Omega)}:=\sup _{\lambda>0} \lambda|\{x \in \Omega:|b(x)|>\lambda\}|^{\frac{1}{2}} .
$$

Note that

$$
\operatorname{div} \frac{x}{|x|^{2}}=2 \pi \delta_{0} \quad \text { in } \quad \mathcal{D}^{\prime}(\Omega)
$$

where $\delta_{0}$ is the delta-function concentrated at $x=0$. We also define the total drift

$$
b^{(\alpha)}:=b-\alpha \frac{x}{|x|^{2}} .
$$

Then from (1.2) we obtain

$$
b^{(\alpha)} \in L_{2, w}(\Omega), \quad\left\|b^{(\alpha)}\right\|_{L_{2, w}(\Omega)} \leq C\left(|\alpha|+\|b\|_{L_{2, w}(\Omega)}\right),
$$

and the divergence of $b^{(\alpha)}$ is sign-definite: $\operatorname{div} b^{(\alpha)} \leq 0$ in the sense of distributions if $\alpha \geq 0$ and $\operatorname{div} b^{(\alpha)}>0$ if $\alpha<0$. The negative $\operatorname{sign}$ of $\operatorname{div} b^{(\alpha)}$ means that the 
drift term generates the positive increase to the quadratic form of our differential operator while the negative sign of $\operatorname{div} b^{(\alpha)}$ means opposite. The goal of this paper is to investigate the existence and properties of weak solutions to the problem (1.1).

Our research is partly motivated by axially symmetric problems for the 3D Navier-Stokes equations. In the study of such problems the equation

$$
\partial_{t} u-\Delta u+\left(v-\alpha \frac{x^{\prime}}{\left|x^{\prime}\right|^{2}}\right) \cdot \nabla u=0 \quad \text { in } \quad \mathbb{R}^{3} \times(0, T)
$$

plays an important role. Here $v=v(x, t)$ is the divergence-free velocity field, $x^{\prime}=$ $\left(x_{1}, x_{2}, 0\right)^{T}$, and $u=u(x, t)$ is some auxiliary scalar function. For example, for axially symmetric solutions without swirl (i.e. if $v(x, t)=v_{r}(r, z, t) e_{r}+v_{z}(r, z, t) e_{z}$ where $e_{r}, e_{\varphi}, e_{z}$ is the standard cylindrical basis) the equation (1.5) is satisfied for $\alpha=2$ and $u=\frac{\omega_{\varphi}}{r}$, where $\omega_{\varphi}:=v_{r, z}-v_{z, r}$ and $r=\left|x^{\prime}\right|$. In the case of general axially symmetric solutions $v(x, t)=v_{r}(r, z, t) e_{r}+v_{\varphi}(r, z, t) e_{\varphi}+v_{z}(r, z, t) e_{z}$ the equation (1.5) holds for $\alpha=-2$ and $u=r v_{\varphi}$.

It is well-known in the Navier-Stokes theory (see, for example, [15, [23, [24, [26], 30]) that while in the case $\alpha>0$ some results like Liouville-type theorems assume no special conditions on the solutions $u$ to the equation (1.5) besides a proper decay of the drift $v$, the analogues results in the case $\alpha<0$ require the additional condition $\left.u\right|_{\Gamma}=0$ where $\Gamma:=\left\{x \in \mathbb{R}^{3}: x_{1}=x_{2}=0\right\}$. Our equation (1.1) can be considered as a $2 \mathrm{D}$ elliptic model for the general equation (1.5) and one of the goals of the present paper is to investigate from the general point of view the role the condition $u(0)=0$ (which is modeling the condition $\left.u\right|_{\Gamma}=0$ in the $3 \mathrm{D}$ situation) plays in the theory.

During the last few decades the problem (1.1) was intensively studied in the case of the divergence-free drift (i.e. for $\alpha=0$ ), see, for example, the papers [5], 6], [11], [18], [19], 21], 25], 27], 29], 31], [32] and references there. Papers devoted to the non-divergence free drifts are not so numerous (see [12], [13, [14 for the references). In the general situation the drift can be decomposed into divergencefree and potential parts. So, our problem (1.1) can also be interpreted as a model problem containing a singular potential part of the drift whose divergence is signdefined.

Our work was motivated to a certain extent by the recent paper [14] where the problem (1.1) was studied in the case of $\Omega \subset \mathbb{R}^{n}$ with $n \geq 3$ and $b \in L_{n, w}(\Omega)$ such that $\operatorname{div} b \in L_{\frac{n}{2}, w}(\Omega), \operatorname{div} b \leq 0$. Our results concerning the case $\alpha \geq 0$ can be viewed as a $2 \mathrm{D}$ version of results in [14]. The main technical difference between [14] and our work is that in the $2 \mathrm{D}$ situation the divergence of the drift is not an integrable function but only a measure.

We define the bilinear form $\mathcal{B}_{\alpha}[u, \eta]$ by

$$
\mathcal{B}_{\alpha}[u, \eta]:=\int_{\Omega} \eta b^{(\alpha)} \cdot \nabla u d x .
$$

This form is well-defined at least for $u \in W_{p}^{1}(\Omega)$ with $p>2$ and $\eta \in L_{q}(\Omega)$ with $q>\frac{2 p}{p-2}$. Note that as $b^{(\alpha)}$ is singular the drift term in (1.6) generally speaking is not integrable for $u \in W_{2}^{1}(\Omega)$. So, instead of the standard notion of weak solutions from the energy class $W_{2}^{1}(\Omega)$ following [14] we introduce the definition of $p$-weak solutions: 
Definition 1.1. Assume $p>2, f \in L_{p}(\Omega)$ and $b$ satisfies (1.2). We say $u$ is a $p$-weak solution to the problem (1.1) if $u \in \stackrel{\circ}{W_{p}^{1}}(\Omega)$ and $u$ satisfies the relation

$$
\int_{\Omega} \nabla u \cdot \nabla \eta d x+\mathcal{B}_{\alpha}[u, \eta]=\int_{\Omega} f \cdot \nabla \eta d x, \quad \forall \eta \in \stackrel{\circ}{W}{ }_{2}^{1}(\Omega) .
$$

From the imbedding theorem we obtain that for $p>2$ every $p$-weak solution to the problem (1.1) is Hölder continuous:

$$
u \in C^{1-\frac{2}{p}}(\bar{\Omega}) .
$$

Moreover, the form $\mathcal{B}_{\alpha}[u, u]$ in (1.7]) possesses the following property.

Proposition 1.1. Assume $b$ satisfies (1.2). Then for any $\alpha \in \mathbb{R}$ and any $v \in \stackrel{\circ}{W_{p}^{1}}(\Omega)$ with $p>2$ the quadratic form $\mathcal{B}_{\alpha}[v, v]$ satisfies the identity

$$
\mathcal{B}_{\alpha}[v, v]=\pi \alpha|v(0)|^{2} .
$$

Proof. Taking into account (1.2) and $\operatorname{div} \frac{x}{|x|^{2}}=0$ in $\Omega \backslash\{0\}$ from (1.6) integrating by parts we obtain

$$
\mathcal{B}_{\alpha}[v, v]=-\lim _{\varepsilon \rightarrow 0} \int_{\Omega \backslash B_{\varepsilon}} \alpha \frac{x}{|x|^{2}} \cdot \nabla \frac{|v|^{2}}{2} d x=\lim _{\varepsilon \rightarrow 0} \frac{\alpha}{2 \varepsilon} \int_{\partial B_{\varepsilon}}|v|^{2} d s=\pi \alpha|v(0)|^{2} .
$$

Here we denote $B_{\varepsilon}:=\left\{x \in \mathbb{R}^{2}:|x|<\varepsilon\right\}$.

To demonstrate effects concerning weak solvability of the problem (1.1) we take $f \equiv 0, b \equiv 0$, and consider the equation

$$
\left\{\begin{array}{c}
-\Delta u-\alpha \frac{x}{|x|^{2}} \cdot \nabla u=0 \quad \text { in } \quad B \\
\left.u\right|_{\partial B}=0
\end{array}\right.
$$

in the unit ball $B:=\left\{x \in \mathbb{R}^{2}:|x|<1\right\}$. For radial solutions $u(x)=v(|x|)$ the equation (1.9) reduces to the ODE for the Darboux-type operator

$$
v^{\prime \prime}(r)+\frac{\alpha+1}{r} v^{\prime}(r)=0, \quad r \in(0,1) .
$$

The family of solutions to this ODE is $v(r)=c_{1} r^{-\alpha}+c_{2}$ if $\alpha \neq 0$ and $c_{1} \ln r+c_{2}$ if $\alpha=0$. If $\alpha \geq 0$ then the only weak radial solution $u(x)=v(|x|)$ to the problem (1.9) such that $u \in W_{2}^{1}(B)$ is identically zero. In contrast, if $\alpha<0$ then we have a one-parameter family $v(x)=c\left(r^{|\alpha|}-1\right)$ of non-trivial $p$-weak solutions to the problem (1.9) with some $p=p(\alpha), p>2$. In particular, for $\alpha<0 p$-weak solutions of the problem (1.9) are non-unique, see also [20] for a related result. Nevertheless, if for $\alpha<0$ we add the additional condition $u(0)=0$ to the problem (1.9) then any radial $p$-weak solution to (1.9) satisfying this requirement is identically zero. This hints that in the case of $\alpha<0$ the condition $u(0)=0$ can serve as an additional requirement that provides uniqueness for the problem (1.1). Our goal is to investigate existence of $p$-weak solutions satisfying this requirement.

The main results of the present paper are the following two theorems: 
Theorem 1.1. Assume $\alpha \geq 0, b$ satisfies (1.2) and $q>2$. Then there exists $p>2$ depending only on $q, \Omega, \alpha$ and $\|b\|_{L_{2, w}(\Omega)}$ such that for any $f \in L_{q}(\Omega)$ there exists a unique p-weak solution $u$ to the problem (1.1). Moreover, this solution satisfies the estimate

$$
\|u\|_{W_{p}^{1}(\Omega)} \leq C\|f\|_{L_{q}(\Omega)},
$$

with the constant $C$ depending only on $q, p, \alpha, \Omega$ and $\|b\|_{L_{2, w}(\Omega)}$.

Theorem 1.2. Assume $\alpha<0, b$ satisfies (1.2) and $q>2$. Then there exists $p>2$ depending only on $q, \Omega, \alpha$ and $\|b\|_{L_{2, w}(\Omega)}$ such that for any $f \in L_{q}(\Omega)$ there exists a unique $p$-weak solution $u$ to the problem (1.1) satisfying the condition $u(0)=0$. Moreover, this solution satisfies the estimate (1.10) with the constant $C$ depending only on $q, p, \alpha, \Omega$ and $\|b\|_{L_{2, w}(\Omega)}$.

Note that the assumption $\partial \Omega$ is of class $C^{1}$ in Theorems 1.1 and 1.2 actually is made only for brevity and our proofs can be extended for more general domains.

Acknowledgement. The research of the second author was supported by RFBR, grant 20-01-00397.

Our paper is organized as follows: in Section 2 we prove some a priori estimates of $p$-weak solutions including higher integrability results, in Section 3 we prove the estimate of the Hölder norm of $p$-weak solutions in the case of $\alpha<0$ which turns out to be a crucial step in our proof of the higher integrability of $\nabla u$ if $\alpha<0$, in Section 4 we present the proofs of Theorems 1.1 and 1.2. in Appendix we recall some known facts and prove an approximation result for divergence-free functions from $L_{2, w}(\Omega)$.

In the paper we use the following notation. For any $a, b \in \mathbb{R}^{n}$ we denote by $a \cdot b$ their scalar product in $\mathbb{R}^{n}$. We denote by $L_{p}(\Omega)$ and $W_{p}^{k}(\Omega)$ the usual Lebesgue and Sobolev spaces. $C_{0}^{\infty}(\Omega)$ is the space of smooth functions compactly supported in $\Omega$. The space $\stackrel{\circ}{W}_{p}^{1}(\Omega)$ is the closure of $C_{0}^{\infty}(\Omega)$ in $W_{p}^{1}(\Omega)$ norm. The space of distributions on $\Omega$ is denoted by $\mathcal{D}^{\prime}(\Omega)$. By $C(\bar{\Omega})$ and $C^{\alpha}(\bar{\Omega}), \alpha \in(0,1)$ we denote the spaces of continuous and Hölder continuous functions on $\bar{\Omega}$. For $q \in[1,+\infty)$ let $L_{q, w}(\Omega)$ be a weak Lebesgue space.

The symbols $\rightarrow$ and $\rightarrow$ stand for the weak and strong convergence respectively. We denote by $B_{R}\left(x_{0}\right)$ the ball in $\mathbb{R}^{n}$ of radius $R$ centered at $x_{0}$ and write $B_{R}$ if $x_{0}=0$. We write also $B$ instead of $B_{1}$. For $\omega \subset \mathbb{R}^{2}$ we denote the average of $f$ over $\omega$ by

$$
(f)_{\omega}:=f_{\omega} f d x=\frac{1}{|\omega|} \int_{\omega} f d x .
$$

For $f, g: \mathbb{R}^{2} \rightarrow \mathbb{R}$ we denote by $f * g$ the convolution

$$
(f * g)(x):=\int_{\mathbb{R}^{2}} f(x-y) g(y) d y .
$$




\section{$2 \quad$ Higher Integrability Results}

In this section we recall a priori estimates for weak solutions to the problem (1.1). Our first theorem is the energy estimate:

Lemma 2.1. Assume $\alpha \in \mathbb{R}$ and $b$ satisfies (1.2). Let $u$ be a p-weak solution to the problem (1.1), corresponding to some $f \in L_{p}(\Omega)$ with $p>2$. In the case of $\alpha<0$ assume additionally that $u(0)=0$. Then the estimate

$$
\|u\|_{W_{2}^{1}(\Omega)} \leq C\|f\|_{L_{2}(\Omega)}
$$

holds with some constant $C>0$ depending only on $\Omega$.

Proof. We take $\eta=u$ in (1.7) and use (1.8).

The next result is the global estimate of $L_{\infty}$-norms of $p$-weak solutions.

Lemma 2.2. Assume $\alpha \in \mathbb{R}$ and b satisfies (1.2). Let $u \in \stackrel{\circ}{W}_{p}^{1}(\Omega)$ be a p-weak solution to the problem (1.1) corresponding to some $f \in L_{q}(\Omega)$ with $q>2$. In the case of $\alpha<0$ assume additionally that $u(0)=0$. Then $u \in L_{\infty}(\Omega)$ and the estimate

$$
\|u\|_{L_{\infty}(\Omega)} \leq C\|f\|_{L_{q}(\Omega)}
$$

holds with some constant $C$ depending only on $\Omega$ and $q$.

Proof. Assume $k \geq 0$ and take $\eta=(u-k)_{+}:=\max \{0, u-k\}$ in (1.7). Note that if $u(0)=0$ and $k \geq 0$ then $(u-k)_{+}(0)=0$. From (1.8) we obtain

$$
\mathcal{B}_{\alpha}\left[u,(u-k)_{+}\right]=\mathcal{B}_{\alpha}\left[(u-k)_{+},(u-k)_{+}\right]=\pi \alpha\left|(u-k)_{+}\right|^{2}(0) .
$$

The last term in non-negative for $\alpha \geq 0$ and vanishes if $\alpha<0, u(0)=0, k \geq 0$. Hence from (1.7) we obtain

$$
\int_{A_{k}}|\nabla u|^{2} d x \leq \int_{A_{k}} f \cdot \nabla u d x, \quad \forall k \geq 0 .
$$

where we denote $A_{k}:=\{x \in \Omega: u(x)>k\}$. The rest of the proof goes as in the usual elliptic theory. Applying the Hölder inequality we obtain

$$
\int_{A_{k}}|\nabla u|^{2} d x \leq\|f\|_{L_{q}(\Omega)}^{2}\left|A_{k}\right|^{\delta}, \quad \forall k \geq 0,
$$

where $\delta:=2\left(\frac{1}{2}-\frac{1}{q}\right)>0$. This inequality yields the following estimate, see [17, Chapter II, Lemma 5.3],

$$
\underset{\Omega}{\operatorname{esssup}} u_{+} \leq C(q, \Omega)\|f\|_{L_{q}(\Omega)}
$$

where $u_{+}:=\max \{0, u\}$. The estimate of esssup $u_{-}$where $u_{-}:=\max \{0,-u\}$ can be obtained in a similar way if we replace $u$ by $^{\Omega}-u$.

Now we prove a higher integrability result for $\nabla u$ in the case of $\alpha \geq 0$. Our proof is based on the reverse Hölder inequality for $\nabla u$, see, for example, 9, Chapter $\mathrm{V}]$. 
Theorem 2.1. Assume $\alpha \geq 0, b$ satisfies (1.2) and $q>2$. Then there exists $p>2$ depending only on $q, \Omega, \alpha$ and $\|b\|_{L_{2, w}(\Omega)}$ such that for any $f \in L_{q}(\Omega)$ and any $p$-weak solution $u$ to the problem (1.1) the estimate (1.10) holds with some constant $C$ depending only on $\Omega, q, \alpha$ and $\|b\|_{L_{2, w}(\Omega)}$.

Proof. First we consider an internal point $x_{0} \in \Omega$. Take $R>0$ such that $B_{2 R}\left(x_{0}\right) \subset$ $\Omega$ and choose a cut-off function $\zeta \in C_{0}^{\infty}\left(B_{2 R}\left(x_{0}\right)\right)$ so that $\zeta \equiv 1$ on $B_{R}\left(x_{0}\right)$ and $\|\nabla \zeta\|_{L_{\infty}\left(\mathbb{R}^{2}\right)} \leq C / R$. Denote $\bar{u}=u-(u)_{B_{2 R}\left(x_{0}\right)}$. Taking $\eta=\zeta^{2} \bar{u}$ in (1.7) and using the relation

$$
\mathcal{B}_{\alpha}\left[u, \zeta^{2} \bar{u}\right]=\mathcal{B}_{\alpha}[\zeta \bar{u}, \zeta \bar{u}]-\int_{B_{2 R}\left(x_{0}\right)} b^{(\alpha)} \cdot \zeta|\bar{u}|^{2} \nabla \zeta d x
$$

after routine computations we arrive at

$$
\begin{aligned}
\int_{B_{R}\left(x_{0}\right)}|\nabla u|^{2} d x+\mathcal{B}_{\alpha}[\zeta \bar{u}, \zeta \bar{u}] & \leq \frac{1}{4} \int_{B_{2 R}\left(x_{0}\right)}|\nabla u|^{2} d x-\int_{B_{2 R}\left(x_{0}\right)} b^{(\alpha)} \cdot \zeta|\bar{u}|^{2} \nabla \zeta d x+ \\
+C & \int_{B_{2 R}\left(x_{0}\right)}\left(|f|^{2}+|\bar{u}|^{2}\right) d x
\end{aligned}
$$

Taking into account Proposition 1.1 and the identity $\zeta(0)=1$ we obtain

$$
\mathcal{B}_{\alpha}[\zeta \bar{u}, \zeta \bar{u}]=\pi \alpha|\bar{u}(0)|^{2}
$$

As $\alpha \geq 0$ we have

$$
\mathcal{B}_{\alpha}[\zeta \bar{u}, \zeta \bar{u}] \geq 0
$$

and hence we can drop this term from (2.3). For the second term on the right-hand side of (2.3) we get

$$
\begin{gathered}
\int_{B_{2 R}\left(x_{0}\right)} b^{(\alpha)} \cdot \zeta|\bar{u}|^{2} \nabla \zeta d x \leq \frac{C}{R}\left\|b^{(\alpha)}\right\|_{L^{2, \infty}}(\Omega)\left\||\bar{u}|^{2}\right\|_{L^{2,1}\left(B_{2 R}\left(x_{0}\right)\right)} \leq \\
\leq \frac{C}{R}\left\|b^{(\alpha)}\right\|_{L^{2, \infty}(\Omega)}\|\bar{u}\|_{L^{4,2}\left(B_{2 R}\left(x_{0}\right)\right)}^{2} .
\end{gathered}
$$

Using the Hölder inequality for Lorentz spaces (see [16, Proposition 2.1], also cited in [22], [8, Section 4.1].) we obtain:

$$
\|\bar{u}\|_{L^{4,2}\left(B_{2 R}\left(x_{0}\right)\right)} \leq C\|1\|_{L^{20,10 / 3}\left(B_{2 R}\left(x_{0}\right)\right)}\|\bar{u}\|_{L^{5,5}\left(B_{2 R}\left(x_{0}\right)\right)} .
$$

Taking into account the property of the indicator function $\left\|\chi_{E}\right\|_{L^{p, s}(\Omega)}=(p / s)^{1 / s} \mid E \cap$ $\left.\Omega\right|^{1 / p}$ we derive

$$
\|\bar{u}\|_{L^{4,2}\left(B_{2 R}\left(x_{0}\right)\right)} \leq C\left|B_{2 R}\right|^{\frac{1}{20}}\|\bar{u}\|_{L_{5}\left(B_{2 R}\left(x_{0}\right)\right)} .
$$

Hence we arrive at

$$
\int_{B_{2 R}\left(x_{0}\right)} b^{(\alpha)} \cdot \zeta|\bar{u}|^{2} \nabla \zeta d x \leq \frac{C}{R^{4 / 5}}\left\|b^{(\alpha)}\right\|_{L_{2, w}(\Omega)}\|\bar{u}\|_{L_{5}\left(B_{2 R}\left(x_{0}\right)\right)}^{2} .
$$


Using the imbedding theorem we estimate

$$
\int_{B_{2 R}\left(x_{0}\right)} b^{(\alpha)} \cdot \zeta|\bar{u}|^{2} \nabla \zeta d x \leq \frac{C}{R^{4 / 5}}\left\|b^{(\alpha)}\right\|_{L_{2, w}(\Omega)}\|\nabla u\|_{L_{\frac{10}{7}}^{2}\left(B_{2 R}\left(x_{0}\right)\right)}^{2} .
$$

Using (2.5) and (2.6) from (2.3) we obtain

$$
\begin{gathered}
\left(\int_{B_{R}\left(x_{0}\right)}|\nabla u|^{2} d x\right)^{\frac{1}{2}} \leq \frac{1}{2}\left(f_{B_{2 R}\left(x_{0}\right)}|\nabla u|^{2} d x\right)^{\frac{1}{2}}+C\left(f_{B_{2 R}\left(x_{0}\right)}|f|^{2} d x\right)^{\frac{1}{2}}+ \\
+C\left(1+\left\|b^{(\alpha)}\right\|_{L_{2, w}(\Omega)}^{1 / 2}\right)\left(f_{B_{2 R}\left(x_{0}\right)}|\nabla u|^{\frac{10}{7}} d x\right)^{\frac{7}{10}} .
\end{gathered}
$$

Now we derive similar estimates near the boundary. Extend $u$ and $f$ by zero outside $\Omega$ and denote this extension by $\tilde{u}$ and $\tilde{f}$. Note that as $\partial \Omega$ is $C^{1}$-smooth there exists $d>0$ depending on $\Omega$ such that for any $R<d$ and any $x \in \partial \Omega$ $\left|B_{R}(x) \backslash \Omega\right|>\frac{1}{4}\left|B_{R}\right|$. Without loss of generality one can assume $d<\operatorname{dist}\{0, \partial \Omega\}$. Take $x_{0} \in \partial \Omega$ and $R<d / 2$. Choose a cut-off function $\zeta \in C_{0}^{\infty}\left(B_{2 R}\left(x_{0}\right)\right)$ so that $\zeta \equiv 1$ on $B_{R}\left(x_{0}\right)$. Then $\zeta^{2} u \in \stackrel{\circ}{W}{ }_{2}^{1}\left(\Omega_{2 R}\left(x_{0}\right)\right)$ where we denote $\Omega_{R}\left(x_{0}\right):=B_{R}\left(x_{0}\right) \cap \Omega$. Taking $\eta=\zeta^{2} u$ in (1.7) after routine computations we arrive at

$$
\int_{B_{R}\left(x_{0}\right)}|\nabla \tilde{u}|^{2} d x+\mathcal{B}_{\alpha}\left[u, \zeta^{2} u\right] \leq \frac{1}{4} \int_{B_{2 R}\left(x_{0}\right)}|\nabla \tilde{u}|^{2} d x+C \int_{B_{2 R}\left(x_{0}\right)}\left(|\tilde{f}|^{2}+|\tilde{u}|^{2}\right) d x
$$

As $0 \notin \Omega_{2 R}\left(x_{0}\right)$ we conclude $\operatorname{div} b^{(\alpha)}=0$ in $\mathcal{D}^{\prime}\left(\Omega_{2 R}\left(x_{0}\right)\right)$ and hence

$$
\mathcal{B}_{\alpha}\left[u, \zeta^{2} u\right]=-\int_{B_{2 R}\left(x_{0}\right)} b^{(\alpha)} \cdot \zeta|\tilde{u}|^{2} \nabla \zeta d x
$$

Repeating the arguments in the internal case we obtain

$$
\int_{B_{2 R}\left(x_{0}\right)} b^{(\alpha)} \cdot \zeta|\tilde{u}|^{2} \nabla \zeta d x \leq \frac{C}{R^{4 / 5}}\left\|b^{(\alpha)}\right\|_{L_{2, w}(\Omega)}\|\tilde{u}\|_{L_{5}\left(B_{2 R}\left(x_{0}\right)\right)}^{2} .
$$

As $\left|\left\{x \in B_{2 R}\left(x_{0}\right): \tilde{u}(x)=0\right\}\right| \geq \frac{1}{4}\left|B_{2 R}\right|$ we can apply Lemma 5.1 and obtain

$$
\|\tilde{u}\|_{L_{5}\left(B_{2 R}\left(x_{0}\right)\right)} \leq C\|\nabla \tilde{u}\|_{L_{\frac{10}{7}}\left(B_{2 R}\left(x_{0}\right)\right)}=C\|\nabla u\|_{L_{\frac{10}{7}}\left(\Omega_{2 R}\left(x_{0}\right)\right)}
$$

with some absolute constant $C>0$. Hence from (2.8) we obtain

$$
\begin{gathered}
\left(\int_{\Omega_{R}\left(x_{0}\right)}|\nabla u|^{2} d x\right)^{\frac{1}{2}} \leq \frac{1}{2}\left(f_{\Omega_{2 R}\left(x_{0}\right)}|\nabla u|^{2} d x\right)^{\frac{1}{2}}+C\left(f_{\Omega_{2 R}\left(x_{0}\right)}|f|^{2} d x\right)^{\frac{1}{2}}+ \\
+C\left(1+\left\|b^{(\alpha)}\right\|_{L_{2, w}(\Omega)}^{1 / 2}\right)\left(f_{\Omega_{2 R}\left(x_{0}\right)}|\nabla u|^{\frac{1}{7}} d x\right)^{\frac{7}{10}}
\end{gathered}
$$


which holds for any $x_{0} \in \partial \Omega$ and any $R<d$. Combining the interior and boundary estimates in the standard way we obtain (2.9) for any $x_{0} \in \bar{\Omega}$ and any $R<d / 2$. This estimate is the reverse Hölder inequality for $\nabla u$, see [9, Chapter V]. Hence there exists $p \in(2, q]$ such that $\nabla u \in L_{p}(\Omega)$ and the following estimate holds:

$$
\|\nabla u\|_{L_{p}(\Omega)} \leq C\left(\|\nabla u\|_{L_{2}(\Omega)}+\|f\|_{L_{q}(\Omega)}\right)
$$

with some constant $C>0$ depending only on $q, \Omega, \alpha$ and $\|b\|_{L_{2, w}(\Omega)}$. Combining this estimate with (2.1) we obtain (1.10).

The estimate of the Hölder norm of solutions to the problem (1.1) is the crucial step in our proof of the higher integrability of $\nabla u$ for $\alpha<0$. This estimate was originally obtained in [2], 3], see also [21]. As the mentioned papers formally deal with a bit stronger assumption on the divergence-free part of the drift $b$ (which in 2D-case corresponds to $b \in L_{2}(\Omega)$ instead of $b \in L_{2, w}(\Omega)$ in our case) we outline the proof of this result.

Theorem 2.2. Assume $\alpha<0, b$ satisfies (1.2) and $q>2$. Then there exists $\mu \in(0,1)$ depending only on $q, \Omega, \alpha$ and $\|b\|_{L_{2, w}(\Omega)}$ such that for any $f \in L_{q}(\Omega)$, any $p>2$ and any $p$-weak solution $u$ to the problem (1.1) the estimate

$$
\|u\|_{C^{\mu}(\bar{\Omega})} \leq C\left(\|f\|_{L_{q}(\Omega)}+\|u\|_{L_{\infty}(\Omega)}\right)
$$

holds with some constant $C$ depending only on $\alpha, \Omega, q$ and $\|b\|_{L_{2, w}(\Omega)}$.

Proof. For any $\alpha \in \mathbb{R}$ the drift $b^{(\alpha)}$ is divergence free in $\Omega \backslash\{0\}$ :

$$
\operatorname{div} b^{(\alpha)}=0 \quad \text { in } \mathcal{D}^{\prime}(\Omega \backslash\{0\}) .
$$

It is well-known that solutions of elliptic equations with the divergence free drifts $b^{(\alpha)} \in L_{2, w}\left(\Omega \backslash B_{R}\right)$ are Hölder continuous, see, for example, [21], 7], 11]. Hence for any $R>0$ such that $B_{2 R} \Subset \Omega$ we have $u \in C^{\mu}\left(\overline{\Omega \backslash B_{R}}\right)$ and for any $x_{0} \in \bar{\Omega} \backslash B_{R}$ and any $0<\rho<R$ the following estimate holds:

$$
\underset{\Omega \cap B_{\rho}\left(x_{0}\right)}{\operatorname{osc}} u \leq C\left(\frac{\rho}{R}\right)^{\mu}\left(\underset{\Omega \cap B_{R}\left(x_{0}\right)}{\operatorname{Osc}} u+\|f\|_{L_{q}\left(\Omega \cap B_{R}\left(x_{0}\right)\right)}\right)
$$

with some constant $\mu \in(0,1)$ and $C>0$ depending only on $q, \Omega, \alpha$ and $\|b\|_{L_{2, w}(\Omega)}$.

On the other hand, for any $0<\rho<R$ we have the estimate

$$
\underset{B_{\rho}}{\operatorname{OSc}} u \leq C\left(\frac{\rho}{R}\right)^{\mu}\left(\underset{B_{R}}{\operatorname{OSc}} u+\|f\|_{L_{q}\left(B_{R}\right)}\right) .
$$

Though this estimate is not new (see, for example [30, Theorem 10.7]), for the reader's convenience we present its proof in the next section, see Theorem 3.1 below. Now take arbitrary $x_{0} \in \Omega$ and denote $r:=\left|x_{0}\right|$. For $r<R$ we obtain $B_{r}\left(x_{0}\right) \subset$ $B_{2 r} \subset B_{2 R}$ and

$$
\begin{aligned}
& \underset{B_{\rho}\left(x_{0}\right)}{\operatorname{Osc}} u \leq C\left(\frac{\rho}{r}\right)^{\mu}\left(\underset{B_{r}\left(x_{0}\right)}{\operatorname{Osc}} u+\|f\|_{L_{q}\left(B_{r}\left(x_{0}\right)\right)}\right) \leq \\
& \leq C\left(\frac{\rho}{r}\right)^{\mu}\left(\underset{B_{2 r}}{\operatorname{asc} u}+\|f\|_{L_{q}\left(B_{2 r}\right)}\right) \leq C\left(\frac{\rho}{r}\right)^{\mu}\left(\frac{r}{R}\right)^{\mu}\left(\underset{B_{2 R}}{\operatorname{Osc} u}+\|f\|_{L_{q}\left(B_{2 R}\right)}\right) \leq \\
& \leq C\left(\frac{\rho}{R}\right)^{\mu}\left(\|u\|_{L_{\infty}(\Omega)}+\|f\|_{L_{q}(\Omega)}\right) \text {. }
\end{aligned}
$$

The last estimate together with (2.11), (2.12) implies (2.10). 
The last theorem of the present section is the analogue of Theorem 2.1 in the case of $\alpha<0$.

Theorem 2.3. Assume $\alpha<0, b$ satisfies (1.2) and $q>2$. Then there exists $p>2$ depending only on $q, \Omega, \alpha$ and $\|b\|_{L_{2, w}(\Omega)}$ such that for any $f \in L_{q}(\Omega)$ and any $p$ weak solution $u$ to the problem (1.1) satisfying the additional assumption $u(0)=0$ the estimate (1.10) holds with some constant $C>0$ depending only on $\Omega, q, \alpha$ and $\|b\|_{L_{2, w}(\Omega)}$. Moreover, without loss of generality one can assume the constant $C$ in (1.10) is a non-decreasing function with respect to $\|b\|_{L_{2, w}(\Omega)}$.

Proof. Assume $\alpha<0$ and $u$ is a $p$-weak solution to the problem (1.1). From Theorem 2.2 we obtain $u \in C^{\mu}(\bar{\Omega})$ with some $\mu \in(0,1)$ and hence integrating by parts and taking into account $u(0)=0$ we obtain

$$
\int_{\Omega} \frac{x}{|x|^{2}} \cdot \nabla u \eta d x=-\int_{\Omega} u \frac{x}{|x|^{2}} \cdot \nabla \eta d x, \quad \forall \eta \in C_{0}^{\infty}(\Omega) .
$$

The last relation means that $u$ is a $p$-weak solution to the problem

$$
\left\{\begin{array}{c}
-\Delta u+b \cdot \nabla u=-\operatorname{div} g \quad \text { in } \Omega, \\
\left.u\right|_{\partial \Omega}=0, \quad u(0)=0,
\end{array}\right.
$$

where

$$
g=f-\alpha \frac{x}{|x|^{2}} u \text {. }
$$

Note that $g \in L_{q_{0}}(\Omega)$ for any $q_{0} \in\left(2, \min \left\{q, \frac{2}{1-\mu}\right\}\right)$ and

$$
\|g\|_{L_{q_{0}}(\Omega)} \leq C\left(\|f\|_{L_{q}(\Omega)}+|\alpha|\|u\|_{C^{\mu}(\bar{\Omega})}\right)
$$

with a constant $C>0$ depending only on $\mu$ and $\Omega$. Applying Theorem 2.1 to the problem (2.14) we conclude there exist constants $p>2$ and $C>0$ depending only on $q_{0}, \Omega$ and $\|b\|_{L_{2, w}(\Omega)}$ such that

$$
\|u\|_{W_{p}^{1}(\Omega)} \leq C\|g\|_{L_{q_{0}}(\Omega)} .
$$

Taking into account (2.15) and (2.10) we obtain the estimate (1.10). 


\section{Hölder Continuity of $p$-Weak Solutions}

The main result of this section is the following estimate:

Proposition 3.1. Assume $\alpha<0$, b satisfies (1.2), $b^{(\alpha)}$ is defined in (1.4). Assume $q>2, f \in L_{q}(\Omega)$ and $B_{2 R} \Subset \Omega$. Assume $p>2$ and $u$ is a $p$-weak solution to

$$
-\Delta u+b^{(\alpha)} \cdot \nabla u=-\operatorname{div} f \quad \text { in } \Omega
$$

such that $u(0)=0$. Then there exist $\mu \in(0,1)$ and $C>0$ depending only on $q, \alpha$, $\|b\|_{L_{2, w}(\Omega)}$ such that for any $0<\rho<R$ the following estimate holds:

$$
\underset{B_{\rho}}{\operatorname{OSc}} u \leq C\left(\frac{\rho}{R}\right)^{\mu}\left(\underset{B_{R}}{\operatorname{OSc}} u+\|f\|_{L_{q}\left(B_{R}\right)}\right) .
$$

Our proof is based on the ideas of [30, Section 10.3], see also [21] where a similar result is obtained. We split the proof of Theorem 3.1 onto several steps. We start from the Caccioppolli-type inequality:

Lemma 3.1. Assume $\alpha<0, b$ satisfies (1.2) in $\Omega=B_{2}$ and $p>2$. Then for any $f \in L_{p}\left(B_{2}\right)$, any $p$-weak solution $u$ to the equation (3.1) in $B_{2}$ such that $u(0) \leq 0$, and any $\zeta \in C_{0}^{\infty}\left(B_{2}\right)$ the following estimate holds:

$$
\int_{B_{2}} \zeta^{2}\left|\nabla u_{+}\right|^{2} d x \leq C \int_{B_{2}}\left|u_{+}\right|^{2}\left(|\nabla \zeta|^{2}+\left|b^{(\alpha)}\right||\nabla \zeta|\right) d x+C \int_{B_{2}} \zeta^{2}|f|^{2} d x .
$$

Here $u_{+}:=\max \{u, 0\}$ and $C>0$ is some absolute constant.

Proof. We take $\eta:=\zeta^{2} u_{+}$in (1.7). The convective term satisfies the identity

$$
\mathcal{B}_{\alpha}\left[u, \zeta^{2} u_{+}\right]=\mathcal{B}_{\alpha}\left[\zeta u_{+}, \zeta u_{+}\right]-\int_{B_{2}} \zeta\left|u_{+}\right|^{2} b^{(\alpha)} \cdot \nabla \zeta d x .
$$

As $u(0) \leq 0$ we obtain $u_{+}(0)=0$ and hence from (1.8) we conclude

$$
\mathcal{B}_{\alpha}\left[\zeta u_{+}, \zeta u_{+}\right]=\pi \alpha \zeta^{2}(0)\left|u_{+}(0)\right|^{2}=0 .
$$

Now the result follows by the Hölder and Young inequalities.

Now we proceed with the following maximum estimate:

Lemma 3.2. Assume $\alpha<0, b$ satisfies (1.2) in $\Omega=B, q>2$ and $f \in L_{q}(B)$. Then there is a constant $C>0$ depending only on $q, \alpha$ and $\|b\|_{L_{2, w}(B)}$ such that for any $p>2$ and any $p$-weak solution $u$ of the equation (3.1) in $B$ satisfying $u(0) \leq 0$ the following estimate holds:

$$
\sup _{B_{1 / 2}} u_{+} \leq C\left(f_{B}\left|u_{+}\right|^{q_{1}} d x\right)^{1 / q_{1}}+C\|f\|_{L_{q}(B)},
$$

where $q_{1}:=\max \left\{5, \frac{2 q}{q-2}\right\}$. 
Proof. The proof of (3.4) follows by application of the standard Moser iteration technique. Let $\beta \geq 0$ be arbitrary and denote

$$
\bar{u}:=u_{+}+k, \quad z:=\bar{u}^{\frac{\beta+2}{2}}, \quad w:=\bar{u}^{\frac{\beta+2}{2}}-k^{\frac{\beta+2}{2}},
$$

where we take $k:=\|f\|_{L_{q}(B)}$ if $f \not \equiv 0$ and $k=0$ otherwise. Assume $\zeta \in C_{0}^{\infty}(B)$ is an arbitrary cut-off function and take $\eta=\zeta^{2} \bar{u}^{\frac{\beta}{2}} w$ in (1.7). We have

$$
\begin{gathered}
\int_{B} \nabla u \cdot \nabla \eta d x \geq \frac{2}{\beta+2} \int_{B} \zeta^{2}|\nabla w|^{2} d x-\frac{4}{\beta+2} \int_{B}|w \nabla \zeta \cdot \zeta \nabla w| d x, \\
\mathcal{B}_{\alpha}[u, \eta]=\frac{2}{\beta+2} \mathcal{B}_{\alpha}\left[w, \zeta^{2} w\right], \\
\int_{B} f \cdot \nabla \eta d x \leq 2 \int_{B} \zeta|f| \bar{u}^{\frac{\beta}{2}}(\zeta|\nabla w|+|\nabla \zeta| w) d x .
\end{gathered}
$$

As $u(0) \leq 0$ we obtain $w(0)=0$. Combining all above inequalities from (1.7) similar to (3.3) we obtain

$$
\int_{B}|\zeta \nabla w|^{2} d x \leq C \int_{B} w^{2}\left(|\nabla \zeta|^{2}+\left|b^{(\alpha)}\right||\nabla \zeta|\right) d x+C(\beta+2)^{2} \int_{B} \zeta^{2}|f|^{2} \bar{u}^{\beta} d x .
$$

Note that $k \leq \bar{u}, w \leq z$ and $\nabla z=\nabla w$. So, in the case $f \not \equiv 0$ from the last inequality we obtain

$$
\int_{B}|\zeta \nabla z|^{2} d x \leq C \int_{B} z^{2}\left(|\nabla \zeta|^{2}+\left|b^{(\alpha)}\right||\nabla \zeta|\right) d x+C(\beta+2)^{2} \int_{B} \zeta^{2}\left|\frac{f}{k}\right|^{2} z^{2} d x .
$$

Recall that $\left\|\frac{f}{k}\right\|_{L_{q}(B)}=1$. Hence the last term can be estimated by the Hölder inequality:

$$
\int_{B} \zeta^{2}\left|\frac{f}{k}\right|^{2} z^{2} d x \leq\left\|\frac{f}{k}\right\|_{L_{q}(B)}^{2}\|\zeta z\|_{L_{\frac{2 q}{q-2}}(B)}^{2} \leq C\|\zeta z\|_{L_{q_{1}}(B)}^{2},
$$

and we arrive at

$$
\int_{B}|\nabla(\zeta z)|^{2} d x \leq C \int_{B} z^{2}\left(|\nabla \zeta|^{2}+\left|b^{(\alpha)}\right||\nabla \zeta|\right) d x+C(\beta+2)^{2}\|\zeta z\|_{L_{q_{1}}(B)}^{2} .
$$

Applying the imbedding theorem

$$
\|\zeta z\|_{L_{2 q_{1}}(B)} \leq C\|\nabla(\zeta z)\|_{L_{2}(B)},
$$

taking arbitrary $\frac{1}{2} \leq r<R \leq 1$, and choosing $\zeta \in C_{0}^{\infty}\left(B_{R}\right)$ so that $\zeta \equiv 1$ on $B_{r}$, $|\nabla \zeta| \leq C /(R-r)$, we get

$$
\int_{B} z^{2}\left|b^{(\alpha)}\right||\nabla \zeta| d x \leq \frac{C}{R-r}\left\|b^{(\alpha)}\right\|_{L_{2, w}\left(B_{R}\right)}\|z\|_{L^{4,2}\left(B_{R}\right)}^{2}
$$

and

$$
\int_{B} z^{2}|\nabla \zeta|^{2} d x \leq\left\||\nabla \zeta|^{2}\right\|_{L_{2, w}\left(B_{R} \backslash B_{r}\right)}\left\|z^{2}\right\|_{L^{2,1}\left(B_{R}\right)} \leq \frac{C}{(R-r)^{3 / 2}}\|z\|_{L^{4,2}\left(B_{R}\right)}^{2} .
$$


Combining these estimates we obtain

$$
\|z\|_{L_{2 q_{1}}\left(B_{r}\right)} \leq \frac{C}{\sqrt{R-r}}\left(\left\|b^{(\alpha)}\right\|_{L_{2, w}(B)}^{\frac{1}{2}}+(R-r)^{-\frac{1}{4}}\right)\|z\|_{L^{4,2}\left(B_{R}\right)}+C(\beta+2)\|z\|_{L_{q_{1}}\left(B_{R}\right)} .
$$

Using the Hölder inequality for Lorentz norms and taking into account $R \in\left(\frac{1}{2}, 1\right]$ we conclude

$$
\|z\|_{L^{4,2}\left(B_{R}\right)} \leq C R^{2\left(1 / 4-1 / q_{1}\right)}\|z\|_{L_{q_{1}}\left(B_{R}\right)} \leq C\|z\|_{L_{q_{1}}\left(B_{R}\right)} .
$$

Applying the Cauchy inequality we derive the estimate

$$
\|z\|_{L_{2 q_{1}}\left(B_{r}\right)} \leq C\left(\beta+2+K(R-r)^{-1}\right)\|z\|_{L_{q_{1}}\left(B_{R}\right)}, \quad K:=\left\|b^{(\alpha)}\right\|_{L_{2, w}(B)}^{\frac{1}{2}}+1,
$$

which is valid for any $\frac{1}{2} \leq r<R \leq 1$. Hence we have

$$
\|\bar{u}\|_{L_{2 \gamma q_{1}}\left(B_{r}\right)} \leq C^{\frac{1}{\gamma}}\left(2 \gamma+K(R-r)^{-1}\right)^{\frac{1}{\gamma}}\|\bar{u}\|_{L_{\gamma q_{1}}\left(B_{R}\right)}
$$

with an arbitrary $\gamma \geq 1, \gamma:=\frac{\beta+2}{2}$. Denote $s_{0}=q_{1}, s_{m}:=2 s_{m-1}$, and denote also $R_{m}=\frac{1}{2}+\frac{1}{2^{m+1}}$. Taking $r=R_{m}, R=R_{m-1}, \gamma=\frac{s_{m-1}}{q_{1}}$ in (3.5) we obtain

$$
\|\bar{u}\|_{L_{s_{m}}\left(B_{R_{m}}\right)} \leq \exp \left(c_{0} m 2^{-m}\right)(1+K)^{2^{1-m}}\|\bar{u}\|_{L_{s_{m-1}}\left(B_{R_{m-1}}\right)} .
$$

Iterating this inequality we get

$$
\|\bar{u}\|_{L_{s_{m}}\left(B_{1 / 2}\right)} \leq C_{0}(1+K)^{2}\|\bar{u}\|_{L_{q_{1}}(B)} .
$$

with some positive constant $C_{0}$ independent on $m$. Taking $m \rightarrow \infty$ we arrive at (3.4).

Lemma 3.3. Assume $\alpha<0, b$ satisfies (1.2) in $\Omega=B, q>2$ and $f \in L_{q}(B)$. Then there are constants $C>0$ and $\varepsilon_{0} \in(0,1)$ depending only on $q, \alpha$ and $\|b\|_{L_{2, w}(B)}$ such that for any $p>2$ and any $p$-weak solution $u$ of the equation (3.1) in $B$ such that $u(0) \leq 0$ if

$$
|\{x \in B: u(x)>0\}| \leq \varepsilon_{0}|B|
$$

then

$$
\sup _{B_{1 / 2}} u_{+} \leq \frac{1}{2} \sup _{B} u_{+}+C\|f\|_{L_{q}(B)}
$$

Proof. From Lemma 3.2 we obtain (3.4) with $q_{1}:=\max \left\{5, \frac{2 q}{q-2}\right\}$. Then we have

$$
C\left(f_{B}\left|u_{+}\right|^{q_{1}} d x\right)^{1 / q_{1}} \leq C \sup _{B} u_{+} \frac{|\{x \in B: u(x)>0\}|^{1 / q_{1}}}{|B|^{1 / q_{1}}} \leq C \varepsilon_{0}^{1 / q_{1}} \sup _{B} u_{+} .
$$

Choosing $C \varepsilon_{0}^{1 / q_{1}}=\frac{1}{2}$ we obtain the required statement.

Next we prove that a small value subset has density less then 1 . 
Lemma 3.4. Assume $\alpha<0, b$ satisfies (1.2) in $\Omega=B$. Then for any $p>2$, any $g \in L_{p}(B)$ and any $p$-weak solution $v$ to the problem

$$
-\Delta v+b^{(\alpha)} \cdot \nabla v=-\operatorname{div} g \quad \text { in } \quad B
$$

satisfying the assumptions

$$
0 \leq v \leq 2 \quad \text { in } \quad B, \quad v(0) \geq 1
$$

if

$$
\|g\|_{L_{2}(B)} \leq c_{\star}|\alpha|, \quad \text { where } \quad c_{\star}:=\frac{\sqrt{\pi}}{4},
$$

then the following estimate holds:

$$
|\{x \in B: v(x)>\lambda\}|>\gamma .
$$

Here $\lambda, \gamma \in(0,1)$ are some constants depending on $\alpha$ and $\|b\|_{L_{3 / 2}(B)}$ in the explicit way described in the proof below.

Proof. Assume there exist $g$ and $v$ satisfying (3.6), (3.7), (3.8) such that

$$
|\{x \in B: v(x)>\lambda\}| \leq \gamma .
$$

Integrating by parts in (1.7) for any $\eta \in C_{0}^{\infty}(B)$ we obtain

$$
2 \pi|\alpha| v(0) \eta(0)=-\int_{B} v\left(\Delta \eta+b^{(\alpha)} \cdot \nabla \eta\right) d x-\int_{B} g \cdot \nabla \eta d x .
$$

Note that $v(0) \geq 1$. Choose $\eta$ so that $\eta=1$ on $B_{1 / 2},\|\nabla \eta\|_{L_{2}(B)} \leq 4 \sqrt{\pi}$ and $\|\eta\|_{C^{2}(\bar{B})} \leq c_{\star \star}$ where $c_{\star \star}>0$ is some sufficiently large absolute constant. Then from (3.8) and the Hölder inequality we obtain

$$
\left|\int_{B} g \cdot \nabla \eta d x\right| \leq \pi|\alpha|
$$

Hence from (3.11) we obtain

$$
\pi|\alpha| \leq\left|\int_{B} v\left(\Delta \eta+b^{(\alpha)} \cdot \nabla \eta\right) d x\right|
$$

Denote

$$
B[v>\lambda]:=\{x \in B: v(x)>\lambda\}, \quad B[v \leq \lambda]:=\{x \in B: v(x) \leq \lambda\} .
$$

From the Hölder inequality we obtain

$$
\int_{B[v>\lambda]} v\left(\Delta \eta+b^{(\alpha)} \cdot \nabla \eta\right) d x \leq\|v\|_{L_{\infty}(B)}\|\eta\|_{C^{2}(\bar{B})}\left(1+\left\|b^{(\alpha)}\right\|_{L_{\frac{3}{2}}(B)}\right)|B[v>\lambda]|^{\frac{1}{3}}
$$

Taking into account (3.7), (3.10) and $\|\eta\|_{C^{2}(B)} \leq c_{\star \star}$ we conclude

$$
\left|\int_{B[v>\lambda]} v\left(\Delta \eta+b^{(\alpha)} \cdot \nabla \eta\right) d x\right| \leq 2 c_{\star \star}\left(1+\left\|b^{(\alpha)}\right\|_{L_{\frac{3}{2}}(B)}\right) \gamma^{\frac{1}{3}}
$$


On the other hand

$$
\left|\int_{B[v \leq \lambda]} v\left(\Delta \eta+b^{(\alpha)} \cdot \nabla \eta\right) d x\right| \leq c_{\star \star}\left(1+\left\|b^{(\alpha)}\right\|_{L_{1}(B)}\right) \lambda .
$$

Finally, we obtain

$$
|\alpha| \leq 2 c_{\star \star}\left(1+\left\|b^{(\alpha)}\right\|_{L_{\frac{3}{2}}(B)}\right) \gamma^{\frac{1}{3}}+c_{\star \star}\left(1+\left\|b^{(\alpha)}\right\|_{L_{1}(B)}\right) \lambda .
$$

This inequality leads to the contradiction if we fix values of $\lambda, \gamma \in(0,1)$ so that

$$
2 c_{\star \star}\left(1+\left\|b^{(\alpha)}\right\|_{L_{\frac{3}{2}}(B)}\right) \gamma^{\frac{1}{3}}+c_{\star \star}\left(1+\left\|b^{(\alpha)}\right\|_{L_{1}(B)}\right) \lambda<\pi|\alpha| .
$$

Lemma 3.5. Assume $\alpha<0, b$ satisfies (1.2) in $\Omega=B_{2}$ and let $\lambda, \gamma \in(0,1)$ be the constants defined in Lemma 3.4. Then for any $\varepsilon>0$ there exists $s_{0} \in \mathbb{N}$ depending only on $\varepsilon, \lambda, \alpha,\|b\|_{L_{2, w}(B)}$ such that for any $p>2$, any $g \in L_{p}(B)$ and any $p$-weak solution $v$ to the problem (3.6) in $B_{2}$ satisfying (3.7) in $B_{2}$ either

$$
\left|\left\{x \in B: v(x) \leq 2^{-s_{0}} \lambda\right\}\right| \leq \varepsilon|B|
$$

or

$$
2^{-s_{0}} \lambda \leq \frac{1}{c_{\star}|\alpha|}\|g\|_{L_{2}\left(B_{2}\right)}
$$

Proof. Assume $s_{0} \in \mathbb{N}$ is arbitrary and $g$ and $v$ satisfy (3.6), (3.7). If

$$
\|g\|_{L_{2}\left(B_{2}\right)} \geq c_{\star}|\alpha|
$$

then (3.14) holds as $\lambda \in(0,1)$. Assume now (3.8) is valid. Then (3.9) is true.

Let $k \in(0,1)$ be arbitrary. Denote $u:=k-v$. Then $u$ satisfies (3.1) and $u(0)=k-v(0) \leq 0$. Then for any $\zeta \in C_{0}^{\infty}\left(B_{2}\right)$ from (3.3) we obtain

$$
\int_{B_{2}} \zeta^{2}\left|\nabla(v-k)_{-}\right|^{2} d x \leq C \int_{B_{2}}(v-k)_{-}^{2}\left(|\nabla \zeta|^{2}+\left|b^{(\alpha)}\right||\nabla \zeta|\right) d x+C \int_{B_{2}}|g|^{2} d x .
$$

Choosing $\zeta$ so that $\zeta \equiv 1$ in $B,|\nabla \zeta| \leq 4$, for any $k \in(0,1)$ we obtain

$$
\int_{B}\left|\nabla(v-k)_{-}\right|^{2} d x \leq C \int_{B_{2}}(v-k)_{-}^{2}\left(1+\left|b^{(\alpha)}\right|\right) d x+C \int_{B_{2}}|g|^{2} d x .
$$

For $s=0,1,2, \ldots$ we denote $k_{s}:=2^{-s} \lambda$ and

$$
A_{s}:=\left\{x \in B: v(x)<k_{s}\right\}=\left\{x \in B:\left(v-k_{s}\right)_{-}(x)>0\right\} .
$$

Applying the De Giorgi inequality (see [17, Chapter II §3, Lemma 3.9]) we obtain

$$
\left(k_{s}-k_{s+1}\right)\left|A_{s+1}\right|^{1 / 2} \leq \frac{C}{\left|B \backslash A_{s}\right|} \int_{A_{s} \backslash A_{s+1}}|\nabla v(x)| d x .
$$

As $B \backslash A_{s} \supset B \backslash A_{0}$ and $\left|B \backslash A_{0}\right| \geq \gamma|B|$ using the Hölder inequality we obtain

$$
2^{-s-1} \lambda\left|A_{s+1}\right|^{1 / 2} \leq \frac{C}{\gamma}\left|A_{s} \backslash A_{s+1}\right|^{1 / 2}\left(\int_{A_{s} \backslash A_{s+1}}|\nabla v|^{2} d x\right)^{1 / 2} .
$$


Hence

$$
\left|A_{s+1}\right| \leq \frac{C}{\lambda^{2} \gamma^{2}} 2^{2 s+2}\left|A_{s} \backslash A_{s+1}\right| \int_{B}\left|\nabla\left(v-k_{s}\right)_{-}\right|^{2} d x .
$$

Using (3.15) with $k=k_{s}$ we arrive at

$$
\left|A_{s+1}\right| \leq C \frac{2^{2 s}}{\lambda^{2} \gamma^{2}}\left|A_{s} \backslash A_{s+1}\right| \int_{B_{2}}\left[\left(v-k_{s}\right)_{-}^{2}\left(1+\left|b^{(\alpha)}\right|\right)+|g|^{2}\right] d x .
$$

As $v \geq 0$ in $B_{2}$ we have $\left(v-k_{s}\right)_{-} \leq k_{s}=2^{-s} \lambda$ in $B_{2}$ and

$$
\left|A_{s+1}\right| \leq \frac{C}{\gamma^{2}}\left|A_{s} \backslash A_{s+1}\right| \int_{B_{2}}\left(1+\left|b^{(\alpha)}\right|+\frac{2^{2 s}}{\lambda^{2}}|g|^{2}\right) d x .
$$

Taking the sum from $s=0$ to $s=s_{0}-1$ and using inclusions $B \supset A_{0} \supset A_{1} \supset \ldots \supset$ $A_{s_{0}}$ we obtain

$$
s_{0}\left|A_{s_{0}}\right| \leq \frac{C_{0}}{\gamma^{2}}\left(1+\left\|b^{(\alpha)}\right\|_{L_{1}\left(B_{2}\right)}+\frac{2^{2 s_{0}}}{\lambda^{2}}\|g\|_{L_{2}\left(B_{2}\right)}^{2}\right)
$$

with some absolute positive constant $C_{0}$. Now let us fix the value $s_{0} \in \mathbb{N}$ so that

$$
\frac{C_{0}}{\gamma^{2} s_{0}}\left(1+c_{\star}^{2}|\alpha|^{2}+\left\|b^{(\alpha)}\right\|_{L_{1}\left(B_{2}\right)}\right) \leq \varepsilon|B|
$$

and consider two cases. If

$$
\frac{2^{s_{0}}}{\lambda}\|g\|_{L_{2}\left(B_{2}\right)} \geq c_{\star}|\alpha|
$$

then (3.14) follows. Otherwise

$$
\frac{2^{s_{0}}}{\lambda}\|g\|_{L_{2}\left(B_{2}\right)} \leq c_{\star}|\alpha|
$$

and from (3.16), (3.17) we obtain (3.13).

Lemma 3.6. Assume $\alpha<0$, $b$ satisfies (1.2) in $\Omega=B_{2}, q>2$ and $g \in L_{q}\left(B_{2}\right)$. Let $\lambda, \gamma \in(0,1)$ be the constants defined in Lemma 3.4. $\varepsilon_{0} \in(0,1)$ be defined in Lemma 3.3 and $s_{0} \in \mathbb{N}$ be the number from Lemma 3.5 defined in (3.17) for $\varepsilon=\varepsilon_{0}$. Denote $\delta:=2^{-s_{0}} \lambda, \delta \in(0,1)$. Then for any $p>2$ and any $p$-weak solution $v$ to the problem (3.6) in $B_{2}$ satisfying (3.7) in $B_{2}$ either

$$
c_{\star}|\alpha| \delta \leq\|g\|_{L_{2}\left(B_{2}\right)}
$$

or

$$
\inf _{B_{1 / 2}} v+c\|g\|_{L_{q}(B)} \geq \frac{\delta}{2} .
$$

Here $c>0$ is some constant depending only on $q, \alpha$ and $\|b\|_{L_{2, w}\left(B_{2}\right)}$. 
Proof. If $g$ satisfies (3.14) then (3.18) follows. Assume now

$$
\|g\|_{L_{2}(B)}<c_{\star}|\alpha| \delta .
$$

Then applying Lemma 3.5 we obtain

$$
|\{x \in B: v(x) \leq \delta\}| \leq \varepsilon_{0}|B| .
$$

Denote $u=\delta-v$. Then $u(0)=\delta-v(0)<0$ and $u$ satisfies all assumptions of Lemma 3.3 with $f=-g$. Hence we obtain

$$
\sup _{B_{1 / 2}}(\delta-v)_{+} \leq \frac{1}{2} \sup _{B}(\delta-v)_{+}+c\|g\|_{L_{q}(B)} \leq \frac{\delta}{2}+c\|g\|_{L_{q}(B)}
$$

which gives

$$
\delta-\inf _{B_{1 / 2}} v \leq \frac{\delta}{2}+c\|g\|_{L_{q}(B)}
$$

Lemma 3.6 is proved.

Lemma 3.7. Assume $\alpha<0, b$ satisfies (1.2) in $\Omega=B_{2}, q>2$ and $f \in L_{q}(B)$. There exist constants $\delta \in(0,1)$ and $c>0$ depending only on $q, \alpha$ and $\|b\|_{L_{2, w}\left(B_{2}\right)}$ such that for any $p>2$ and any a p-weak solution $u$ to the equation (3.1) in $B_{2}$ satisfying $u(0)=0$ the following inequality holds:

$$
\underset{B_{1 / 2}}{\operatorname{OSc}} u \leq\left(1-\frac{\delta}{2}\right) \underset{B_{2}}{\operatorname{Oosc}} u+c\|f\|_{L_{q}\left(B_{2}\right)} .
$$

Proof. For any $r \in(0,2)$ we denote

$$
M(r):=\sup _{B_{r}} u, \quad m(r):=\inf _{B_{r}} u, \quad \omega(r):=M(r)-m(r),
$$

Note that $m(2) \leq 0 \leq M(2)$. Denote $k_{0}:=\frac{m(2)+M(2)}{2}$, and define $v$ and $g$ so that

$$
\begin{aligned}
& v(x):=2 \frac{u(x)-m(2)}{\omega(2)}, \quad g(x):=\frac{2 f(x)}{\omega(2)}, \quad \text { if } \quad k_{0}<0, \\
& v(x):=2 \frac{M(2)-u(x)}{\omega(2)}, \quad g(x):=-\frac{2 f(x)}{\omega(2)}, \quad \text { if } \quad k_{0} \geq 0 .
\end{aligned}
$$

Then $v$ is a $p$-weak solution to the equation (3.6) in $B_{2}$ satisfying the additional conditions (3.7) in $B_{2}$. From Lemma 3.6 we conclude

$$
\text { either } \quad \delta \leq c\|g\|_{L_{2}\left(B_{2}\right)} \quad \text { or } \quad \inf _{B_{1 / 2}} v+c\|g\|_{L_{q}(B)} \geq \frac{\delta}{2} .
$$

In the first case from the Hölder inequality we obtain

$$
\omega(2) \leq \frac{c}{\delta}\|f\|_{L_{2}\left(B_{2}\right)} \leq c(\delta, q)\|f\|_{L_{q}\left(B_{2}\right)}
$$

and hence (3.20) follows. In the second case if $k_{0}<0$ we have

$$
m(1 / 2)-m(2)+2 c\|f\|_{L_{q}(B)} \geq \frac{\delta}{2} \omega(2)
$$


and hence

$$
\left(1-\frac{\delta}{2}\right) \omega(2)+2 c\|f\|_{L_{q}(B)} \geq \omega(1 / 2)
$$

and we obtain (3.20). If $k_{0} \geq 0$ then

$$
M(2)-M(1 / 2)+2 c\|f\|_{L_{q}(B)} \geq \frac{\delta}{2} \omega(2)
$$

and we again obtain (3.20).

Lemma 3.8. Assume $\alpha<0, b$ satisfies (1.2) in $\Omega$. Assume $q>2, f \in L_{q}(\Omega)$ and $B_{2 R} \Subset \Omega$. Then there exist constants $\delta \in(0,1)$ and $c>0$ depending only on $q, \alpha$ and $\|b\|_{L_{2, w}\left(B_{2 R}\right)}$ such that for any $p>2$ and any $p$-weak solution $u$ to the equation (3.1) in $\Omega$ satisfying $u(0)=0$ the following inequality holds:

$$
\underset{B_{R / 2}}{\operatorname{OSc}} u \leq\left(1-\frac{\delta}{2}\right) \underset{B_{2 R}}{\operatorname{OSc}} u+c R^{1-\frac{2}{q}}\|f\|_{L_{q}\left(B_{2 R}\right)} .
$$

Proof. For $x \in B_{2}$ we denote

$$
u^{R}(x)=u(R x), \quad b_{R}^{(\alpha)}(x)=R b^{(\alpha)}(R x), \quad f^{R}(x)=R f(R x) .
$$

Then $u^{R}$ is a solution to

$$
-\Delta u^{R}+b_{R}^{(\alpha)} \cdot \nabla u^{R}=-\operatorname{div} f^{R} \quad \text { in } \quad B_{2}
$$

and, moreover,

$$
\left\|b_{R}^{(\alpha)}\right\|_{L_{2, w}\left(B_{2}\right)}=\left\|b^{(\alpha)}\right\|_{L_{2, w}\left(B_{2 R}\right)}, \quad\left\|f^{R}\right\|_{L_{q}\left(B_{2}\right)}=R^{1-\frac{2}{q}}\|f\|_{L_{q}\left(B_{2 R}\right)} .
$$

From Lemma 3.7 we obtain

$$
\underset{B_{1 / 2}}{\operatorname{OSc}} u^{R} \leq\left(1-\frac{\delta}{2}\right) \underset{B_{2}}{\operatorname{Oosc}} u^{R}+c\left\|f^{R}\right\|_{L_{q}\left(B_{2}\right)}
$$

which implies (3.21).

Now the inequality (3.2) follows from (3.21) by the standard iteration technique. Proposition 3.1 is proved. 


\section{Proof of Main Results}

We start with the proof of Theorem 1.1. To construct a $p$-weak solution to the problem (1.1) we approximate our $\operatorname{drift} b^{(\alpha)}$ by smooth functions using Lemma 4.2. In the case $\alpha \geq 0$ our construction shows that $p$-weak solutions are always approximative solutions in the sense they can be obtained as limits of smooth solutions of the equations with smooth drifts. Note that this is not true if $\alpha<0$ as the approximative solution must satisfy the maximum principle (locally), but the radial solutions constructed in the case of $\alpha<0$ in Section 1 do not possess this property.

Proposition 4.1. Assume $\alpha \geq 0, b \in C^{\infty}(\bar{\Omega})$ satisfies $\operatorname{div} b=0$ in $\Omega$ and $q>2$. Assume $f \in L_{q}(\Omega)$ and $\varepsilon>0$. Define

$$
b_{\varepsilon}^{(\alpha)}:=b-\alpha \frac{x}{|x|^{2}+\varepsilon^{2}} .
$$

Then there exists a unique q-weak solution $u$ to the problem

$$
\left\{\begin{array}{c}
-\Delta u+b_{\varepsilon}^{(\alpha)} \cdot \nabla u=-\operatorname{div} f \quad \text { in } \Omega, \\
\left.u\right|_{\partial \Omega}=0 .
\end{array}\right.
$$

Moreover, there exists $p \in(2, q]$ depending only on $q, \Omega, \alpha$ and $\|b\|_{L_{2, w}(\Omega)}$ such that the following estimate holds:

$$
\|u\|_{W_{p}^{1}(\Omega)} \leq C\|f\|_{L_{q}(\Omega)} .
$$

Here $C>0$ is a constant which can be chosen to be a non-decreasing function of $q$, $\Omega, \alpha$ and $\|b\|_{L_{2, w}(\Omega)}$.

Proof. The existence and uniqueness of the weak solution $u \in W_{2}^{1}(\Omega)$ for equation (4.1) with the smooth drift $b_{\varepsilon}^{(\alpha)}$ is well-known, see, for example, [17. Moreover, from $L_{q}$-theory for equations with smooth coefficients we obtain $u^{\varepsilon} \in W_{q}^{1}(\Omega)$. Note that as $\alpha \geq 0$ we have $\operatorname{div} b_{\varepsilon}^{(\alpha)} \leq 0$ in $\Omega$. Hence the estimate (4.2) follows by the arguments similar to the proof of Theorem 2.1.

Now we state an approximation result in weak Lebesgue space:

Proposition 4.2. Let $\Omega \subset \mathbb{R}^{2}$ be a bounded simply connected domain of class $C^{1}$. Assume $b \in L_{2, w}(\Omega), \operatorname{div} b=0$ in $\mathcal{D}^{\prime}(\Omega)$. Then there exist $b^{\varepsilon} \in C^{\infty}(\bar{\Omega}), \operatorname{div} b^{\varepsilon}=0$ in $\mathcal{D}^{\prime}(\Omega)$, such that

1) $\left\|b^{\varepsilon}\right\|_{L_{2, w}(\Omega)} \leq C\|b\|_{L_{2, w}(\Omega)}$,

2) $b^{\varepsilon} \rightarrow b$ a.e. in $\Omega$ as $\varepsilon \rightarrow 0$.

Here the constant $C>0$ depends only on $\Omega$.

Proof. Assume $p \in(1,+\infty)$ and denote

$$
J_{p}(\Omega):=\left\{u \in L_{p}\left(\Omega ; \mathbb{R}^{2}\right): \operatorname{div} u=0 \text { in } \mathcal{D}^{\prime}(\Omega)\right\} .
$$

Consider any bounded simply connected domain $\Omega_{0} \subset \mathbb{R}^{2}$ such that $\Omega \Subset \Omega_{0}$. Then there exists an extension operator $T: J_{p}(\Omega) \rightarrow J_{p}\left(\mathbb{R}^{2}\right)$ such that for all $b \in J_{p}(\Omega)$ the function $\tilde{b}:=T b$ has the following properties

$$
\begin{aligned}
& \tilde{b} \equiv 0 \quad \text { in } \quad \mathbb{R}^{2} \backslash \Omega_{0},\left.\quad \tilde{b}\right|_{\Omega}=b \\
& \operatorname{div} \tilde{b}=0 \quad \text { in } \quad \mathcal{D}^{\prime}\left(\mathbb{R}^{2}\right), \quad\|\tilde{b}\|_{L_{p}\left(\mathbb{R}^{2}\right)} \leq c_{p}\|b\|_{L_{p}(\Omega)} .
\end{aligned}
$$


The existence of the extension operator $T$ is shown, for example, in [10, $\S 3.1$, Theorem 3.1], and $L_{p}$-estimates for this operator follow from the corresponding estimates for weak solutions to the Neumann problem involved into the construction of the operator $T$. Indeed, denote by $E: W_{p^{\prime}}^{1}\left(\Omega_{0} \backslash \Omega\right) \rightarrow W_{p^{\prime}}^{1}\left(\Omega_{0}\right)$ any bounded linear extension operator, i.e. $\left.(E \eta)\right|_{\Omega_{0} \backslash \Omega}=\eta$. Then the formula

$$
l(\eta):=-\int_{\Omega} b \cdot \nabla \tilde{\eta} d x, \quad \tilde{\eta}:=E \eta, \quad \eta \in W_{p^{\prime}}^{1}\left(\Omega_{0} \backslash \Omega\right)
$$

determines a bounded linear functional on the Banach space $W_{p^{\prime}}^{1}\left(\Omega_{0} \backslash \Omega\right)$. Moreover, the condition $\operatorname{div} b=0$ in $\mathcal{D}^{\prime}(\Omega)$ implies that the functional $l$ is independent of the extension operator $E$. Hence there exists a unique solution $\varphi \in W_{p}^{1}\left(\Omega_{0} \backslash \Omega\right)$ of the Neumann problem

$$
\begin{aligned}
& \int_{\Omega_{0} \backslash \Omega} \nabla \varphi \cdot \nabla \eta d x=l(\eta), \quad \forall \eta \in W_{p^{\prime}}^{1}\left(\Omega_{0} \backslash \Omega\right), \\
& \int_{\Omega_{0} \backslash \Omega} \varphi d x=0, \quad\|\nabla \varphi\|_{L_{p}\left(\Omega_{0} \backslash \Omega\right)} \leq c\|b\|_{L_{p}(\Omega)},
\end{aligned}
$$

and one can take $\tilde{b}:=b$ in $\Omega$ and $\tilde{b}:=\nabla \varphi$ in $\Omega_{0} \backslash \Omega$ and then extend $\tilde{b}$ by zero to the whole $\mathbb{R}^{2}$. It is easy to see that $\tilde{b} \in J_{p}\left(\mathbb{R}^{2}\right)$ and $T b:=\tilde{b}$ satisfies all necessary properties.

From the Marcinkiewicz interpolation theorem (see [8, Theorem 1.4.19]) we conclude that $T$ is also bounded as an operator from $L_{2, w}(\Omega)$ into $L_{2, w}\left(\mathbb{R}^{2}\right)$ :

$$
\|\tilde{b}\|_{L_{2, w}\left(\mathbb{R}^{2}\right)} \leq c_{2, w}\|b\|_{L_{2, w}(\Omega)}, \quad \forall b \in L_{2, w}(\Omega): \quad \operatorname{div} b=0 \quad \text { in } \quad \mathcal{D}^{\prime}(\Omega) .
$$

Let $\omega_{\varepsilon}$ be the standard Sobolev kernel $\omega_{\varepsilon}(x)=\varepsilon^{-2} \omega(x / \varepsilon), \omega \in C_{0}^{\infty}(B), \int_{\mathbb{R}^{2}} \omega(x) d x=$ 1 , and denote

$$
\tilde{b}^{\varepsilon}:=\omega_{\varepsilon} * \tilde{b}, \quad b^{\varepsilon}:=\left.\tilde{b}^{\varepsilon}\right|_{\Omega} .
$$

Then for $b \in L_{2, w}(\Omega)$ we have

$\tilde{b}^{\varepsilon} \in C_{0}^{\infty}\left(\mathbb{R}^{2}\right), \quad \operatorname{div} \tilde{b}^{\varepsilon}=0 \quad$ in $\quad \mathcal{D}^{\prime}\left(\mathbb{R}^{2}\right), \quad \tilde{b}^{\varepsilon} \rightarrow \tilde{b} \quad$ in $\quad L_{p}\left(\mathbb{R}^{2}\right), \quad \forall p \in[1,2)$.

Moreover, as the convolution operator $T_{\varepsilon} \tilde{b}:=\omega_{\varepsilon} * \tilde{b} \equiv \tilde{b}^{\varepsilon}$ is bounded in $L_{p}\left(\mathbb{R}^{2}\right)$ for any $p \in(1,+\infty)$ with $\left\|T_{\varepsilon}\right\|_{L_{p} \rightarrow L_{p}}=1$, i.e.

$$
\left\|\tilde{b}^{\varepsilon}\right\|_{L_{p}\left(\mathbb{R}^{2}\right)} \leq\|\tilde{b}\|_{L_{p}\left(\mathbb{R}^{2}\right)}, \quad \forall p \in(1,+\infty),
$$

from the Marcinkiewicz interpolation theorem (see [8, Theorem 1.4.19]) we conclude that $T_{\varepsilon}$ is also bounded as an operator from $L_{2, w}\left(\mathbb{R}^{2}\right)$ into $L_{2, w}\left(\mathbb{R}^{2}\right)$ and the norm $\left\|T_{\varepsilon}\right\|_{L_{2, w} \rightarrow L_{2, w}}$ is independent of $\varepsilon$ :

$$
\exists M>0: \quad\left\|\tilde{b}^{\varepsilon}\right\|_{L_{2, w}\left(\mathbb{R}^{2}\right)} \leq M\|\tilde{b}\|_{L_{2, w}\left(\mathbb{R}^{2}\right)} .
$$

Hence $b^{\varepsilon}$ possesses all required properties.

Now we can prove Theorem 1.1: 
Proof. Let $p>2$ be the exponent defined in Proposition 4.1. Note that $p$ depends only on $q, \Omega, \alpha$ and $\|b\|_{L_{2, w}(\Omega)}$. From Proposition 4.2 we obtain the existence of a sequence $b_{\varepsilon} \in C^{\infty}(\bar{\Omega})$ such that

$$
\operatorname{div} b_{\varepsilon}=0 \quad \text { in } \quad \Omega, \quad b_{\varepsilon} \rightarrow b \quad \text { a.e. in } \quad \Omega, \quad\left\|b_{\varepsilon}\right\|_{L_{2, w}(\Omega)} \leq C\|b\|_{L_{2, w}(\Omega)} .
$$

Denote

$$
b_{\varepsilon}^{(\alpha)}:=b_{\varepsilon}-\alpha \frac{x}{|x|^{2}+\varepsilon^{2}}
$$

and note that

$$
\left\|b_{\varepsilon}^{(\alpha)}\right\|_{L_{2, w}(\Omega)} \leq c\left(|\alpha|+\|b\|_{L_{2, w}(\Omega)}\right) .
$$

From Proposition 4.1 we conclude that for any $\varepsilon>0$ there exists a $q$-weak solution $u^{\varepsilon}$ to the problem (4.1) (with $b_{\varepsilon}^{(\alpha)}$ defined in (4.4)). Moreover, as the constant $C$ in (4.2) is a non-decreasing function of $\|b\|_{L_{2, w}(\Omega)}$ from (4.5) we conclude that the estimate

$$
\left\|u^{\varepsilon}\right\|_{W_{p}^{1}(\Omega)} \leq C\|f\|_{L_{q}(\Omega)}
$$

holds with some constant $C>0$ independent on $\varepsilon$. From (4.6) we obtain existence of $u \in W_{p}^{1}(\Omega)$ such that for some subsequence $u^{\varepsilon}$ we have

$$
u^{\varepsilon} \rightarrow u \quad \text { in } W_{p}^{1}(\Omega) .
$$

On the other hand, from (4.3) we conclude there exists a subsequence such that

$$
b_{\varepsilon}^{(\alpha)} \rightarrow b^{(\alpha)} \quad \text { in } \quad L_{p^{\prime}}(\Omega), \quad p^{\prime}=\frac{p}{p-1} .
$$

Hence for any $\eta \in C_{0}^{\infty}(\Omega)$ from (4.7) and (4.8) we obtain

$$
\int_{\Omega} b_{\varepsilon}^{(\alpha)} \cdot \nabla u^{\varepsilon} \eta d x \rightarrow \int_{\Omega} b^{(\alpha)} \cdot \nabla u \eta d x
$$

as $\varepsilon \rightarrow 0$. Passing to the limit in the equations for $u^{\varepsilon}$ (in a weak form) we obtain (1.7). Hence $u$ is a $p$-weak solution to the problem (1.1). The uniqueness of $p$-weak solutions follows from (2.1).

Now we present a proof of Theorem [1.2. We split it into several steps.

Lemma 4.1. Assume $\alpha<0$. Then there exists $p_{1}>2$ depending only on $\alpha$ and $\Omega$ such that for any $B_{\varepsilon} \Subset \Omega$ and any $f^{\varepsilon} \in C_{0}^{\infty}\left(\Omega \backslash B_{\varepsilon}\right)$ there exists a unique $p_{1}$-weak solution $w^{\varepsilon} \in \stackrel{\circ}{W} p_{p_{1}}^{1}(\Omega)$ to the problem

$$
\left\{\begin{aligned}
-\Delta w^{\varepsilon}-|\alpha| \frac{x}{|x|^{2}} \cdot \nabla w^{\varepsilon} & =-|x|^{\alpha} \operatorname{div} f^{\varepsilon} \quad \text { in } \quad \mathcal{D}^{\prime}(\Omega), \\
\left.w^{\varepsilon}\right|_{\partial \Omega} & =0 .
\end{aligned}\right.
$$

Proof. As the right-hand side of the equation (4.9) is smooth it can be represented in the form $\operatorname{div} g^{\varepsilon}$ for some $g^{\varepsilon} \in L_{q_{1}}(\Omega)$ with some $q_{1}>2$. Hence the result follows from Theorem 1.1 .

Lemma 4.2. Assume $\alpha<0$. Then there exists $p_{2}>2$ depending only on $\alpha$ and $\Omega$ such that for any $B_{\varepsilon} \Subset \Omega$ and any $f^{\varepsilon} \in C_{0}^{\infty}\left(\Omega \backslash B_{\varepsilon}\right)$ there exists a unique $p_{2}$-weak solution $u^{\varepsilon}$ to the problem

$$
\left\{\begin{array}{c}
-\Delta u^{\varepsilon}-\alpha \frac{x}{|x|^{2}} \cdot \nabla u^{\varepsilon}=-\operatorname{div} f^{\varepsilon} \quad \text { in } \quad \mathcal{D}^{\prime}(\Omega), \\
\left.u^{\varepsilon}\right|_{\partial \Omega}=0, \quad u^{\varepsilon}(0)=0 .
\end{array}\right.
$$


Proof. Let $w^{\varepsilon}$ be a unique $p_{1}$-weak solution to the problem (4.9) with some $p_{1}>2$ depending only on $\alpha$ and $\Omega$. Define the function

$$
u^{\varepsilon}(x):=|x|^{|\alpha|} w^{\varepsilon}(x) .
$$

It is easy to see that $u^{\varepsilon} \in W_{p_{2}}^{1}(\Omega)$ for some $p_{2}>2$ depending only on $p_{1}$ and $|\alpha|$. The direct computation shows that $u^{\varepsilon}$ is a $p_{2}$-weak solution to the problem (4.10).

Lemma 4.3. Assume $\alpha<0$ and $q>2$. There exists $p>2$ depending only on $q$, $\Omega$ and $\alpha$ such that for any $f \in L_{q}(\Omega)$ there exists a unique $p$-weak solution $u$ to the problem

$$
\left\{\begin{array}{c}
-\Delta u-\alpha \frac{x}{|x|^{2}} \cdot \nabla u=-\operatorname{div} f \quad \text { in } \Omega, \\
\left.u\right|_{\partial \Omega}=0, \quad u(0)=0 .
\end{array}\right.
$$

Moreover, $u$ satisfies the estimate

$$
\|u\|_{W_{p}^{1}(\Omega)} \leq C\|f\|_{L_{q}(\Omega)}
$$

where $C>0$ is some constant depending only on $q$ and $\Omega$.

Proof. Assume $f \in L_{q}(\Omega)$ and take $f^{\varepsilon} \in C_{0}^{\infty}\left(\Omega \backslash B_{\varepsilon}\right)$ so that $f^{\varepsilon} \rightarrow f$ in $L_{q}(\Omega)$ as $\varepsilon \rightarrow+0$. Denote by $p>2$ the exponent determined in Theorem 2.3 (without loss of generality we can assume $p \leq p_{2}$ where $p_{2}$ is determined in Lemma 4.2). Denote by $u^{\varepsilon}$ a unique $p$-weak solution to the problem (4.10). Then from Theorem 2.3 we obtain

$$
\left\|u^{\varepsilon}\right\|_{L_{p}(\Omega)} \leq C\left\|f^{\varepsilon}\right\|_{L_{q}(\Omega)}
$$

and hence there exists $u \in \stackrel{\circ}{W}_{p}^{1}(\Omega)$ such that for some subsequence the weak convergence $u^{\varepsilon} \rightarrow u$ in $W_{p}^{1}(\Omega)$ takes place. It is easy to see that $u$ satisfies the integral identity (1.7) with $b=0$. Moreover, as $u^{\varepsilon}(0)=0$ and the imbedding $W_{p}^{1}(\Omega) \hookrightarrow C(\bar{\Omega})$ is compact we obtain $u(0)=0$. So, $u$ is a $p$-weak solution to the problem (4.11). The uniqueness of $u$ follows from (2.1).

Lemma 4.4. Assume $\alpha<0, b \in C^{\infty}(\bar{\Omega})$, $\operatorname{div} b=0$ in $\Omega, q>2$ and $p>2$ is the exponent depending only on $\Omega, q, \alpha,\|b\|_{L_{2, w}(\Omega)}$ defined in Lemma 4.3. Then for any $f \in L_{q}(\Omega), v \in L_{q}(\Omega)$ there exists a unique $p$-weak solution $u \in \stackrel{\circ}{W_{p}^{1}}(\Omega)$ to the problem

$$
\left\{\begin{array}{c}
-\Delta u-\alpha \frac{x}{|x|^{2}} \cdot \nabla u=-\operatorname{div}(f+b v) \quad \text { in } \quad \Omega, \\
\left.u\right|_{\partial \Omega}=0, \quad u(0)=0 .
\end{array}\right.
$$

Moreover, the operator $A: L_{q}(\Omega) \rightarrow L_{q}(\Omega), A(v):=u$, is continuous and compact.

Proof. For given $f, v \in L_{q}(\Omega)$ the existence of a unique $p$-weak solution $u$ satisfying $u(0)=0$ follows from Lemma 4.3. Moreover, $u$ satisfies the estimate

$$
\|u\|_{W_{p}^{1}(\Omega)} \leq C\left(\|f\|_{L_{q}(\Omega)}+\|b\|_{L_{\infty}(\Omega)}\|v\|_{L_{q}(\Omega)}\right) .
$$

For a given $f \in L_{q}(\Omega)$ the last estimate implies

$$
\left\|A\left(v_{1}\right)-A\left(v_{2}\right)\right\|_{W_{p}^{1}(\Omega)} \leq C\|b\|_{L_{\infty}(\Omega)}\left\|v_{1}-v_{2}\right\|_{L_{q}(\Omega)}
$$

and hence the operator $A$ is continuous as an operator from $L_{q}(\Omega)$ into $L_{q}(\Omega)$. The compactness of this operator in $L_{q}(\Omega)$ follows from the compactness of the imbedding of $W_{p}^{1}(\Omega)$ into $L_{q}(\Omega)$. 
Lemma 4.5. Assume $\alpha<0, b \in C^{\infty}(\bar{\Omega}), \operatorname{div} b=0$ in $\Omega, q>2$ and $p>2$ is the exponent depending only on $\Omega, q, \alpha,\|b\|_{L_{2, w}(\Omega)}$ defined in Lemma 4.3. Then for any $f \in L_{q}(\Omega)$ there exists a unique p-weak solution $u$ to the problem

$$
\left\{\begin{aligned}
-\Delta u+\left(b-\alpha \frac{x}{|x|^{2}}\right) \cdot \nabla u & =-\operatorname{div} f \quad \text { in } \quad \Omega, \\
\left.u\right|_{\partial \Omega}=0, \quad u(0) & =0 .
\end{aligned}\right.
$$

Proof. We apply the Leray-Schauder fixed point theorem for the operator $A$ : $L_{q}(\Omega) \rightarrow L_{q}(\Omega)$ defined in Lemma 4.4. Assume $\lambda \in[0,1]$ and $v \in L_{q}(\Omega)$ satisfies $v=\lambda A(v)$. Denote $u:=A(v)$. Then $u$ is a unique $p$-weak solution to the problem

$$
\left\{\begin{array}{c}
-\Delta u+\left(\lambda b-\alpha \frac{x}{|x|^{2}}\right) \cdot \nabla u=-\operatorname{div} f \quad \text { in } \quad \Omega, \\
\left.u\right|_{\partial \Omega}=0, \quad u(0)=0 .
\end{array}\right.
$$

From Theorem 2.3 we obtain the estimate

$$
\|u\|_{W_{p}^{1}(\Omega)} \leq C\|f\|_{L_{q}(\Omega)}
$$

with some constant depending only on $q, \Omega, \alpha$ and $\|b\|_{L_{2, w}(\Omega)}$ and independent of $\lambda \in[0,1]$. Hence there exists $u \in \stackrel{\circ}{W} \underset{p}{1}(\Omega)$ satisfying $u=A(u)$.

Finally, we can relax the smoothness conditions on the divergence-free part of the drift and prove Theorem 1.2 ,

Proof. Assume $b$ satisfies (1.2) and let $p>2$ be the exponent defined in Lemma 4.5. From Lemma 4.2 we obtain existence of $b_{\varepsilon} \in C^{\infty}(\bar{\Omega})$ such that $\operatorname{div} b_{\varepsilon}=0$ in $\Omega,\left\|b_{\varepsilon}\right\|_{L_{2, w}(\Omega)} \leq c\|b\|_{L_{2, w}(\Omega)} \leq$ and $b_{\varepsilon} \rightarrow b$ in $L_{p^{\prime}}(\Omega)$ where $p^{\prime}=\frac{p}{p-1}$. From Lemma 4.5 we conclude that for any $\varepsilon>0$ there exists a unique $p$-weak solution $u^{\varepsilon}$ to the problem

$$
\left\{\begin{array}{l}
-\Delta u^{\varepsilon}+b_{\varepsilon}^{(\alpha)} \cdot \nabla u^{\varepsilon}=-\operatorname{div} f \quad \text { in } \Omega, \\
\left.u^{\varepsilon}\right|_{\partial \Omega}=0, \quad u^{\varepsilon}(0)=0,
\end{array}\right.
$$

where $b_{\varepsilon}^{(\alpha)}=b_{\varepsilon}-\alpha \frac{x}{|x|^{2}}$. From Theorem 2.3 we obtain the estimate

$$
\left\|u^{\varepsilon}\right\|_{W_{p}^{1}(\Omega)} \leq C\|f\|_{L_{q}(\Omega)}
$$

where the constant $C>0$ depends only on $a, \Omega, \alpha$ and $\|b\|_{L_{2, w}(\Omega)}$. Then there exists $u \in \stackrel{\circ}{W}{ }_{p}^{1}(\Omega)$ such that for some subsequence $u^{\varepsilon}$ the convergence (4.7) holds. The rest of the proof repeats the proof of Theorem 1.1 in the beginning of this section. The identity $u(0)=0$ follows from $u^{\varepsilon}(0)=0$ and compactness of the imbedding of $W_{p}^{1}(\Omega)$ into $C(\bar{\Omega})$. The uniqueness of $p$-weak solutions follows from (2.1). 


\section{Appendix}

Here we present a variant of the imbedding theorem that we used in Section 2

Proposition 5.1. Assume $B_{R} \subset \mathbb{R}^{n}, n \geq 2, p \in[1, n)$ and $p_{*}:=\frac{p n}{n-p}$. Then for any $\lambda \in(0,1)$ there exists $C=C(n, p)>0$ such that if $u \in W_{p}^{1}\left(B_{R}\right)$ satisfies

$$
\left|\left\{x \in B_{R}: u(x)=0\right\}\right| \geq \lambda\left|B_{R}\right|
$$

then

$$
\|u\|_{L_{p_{*}}\left(B_{R}\right)} \leq C(n, p) \lambda^{-1}\|\nabla u\|_{L_{p}\left(B_{R}\right)} .
$$

Proof. From the Sobolev imbedding theorem we conclude

$$
\|u\|_{L_{p_{*}}\left(B_{R}\right)} \leq \frac{c}{R}\|u\|_{L_{p}\left(B_{R}\right)}+c\|\nabla u\|_{L_{p}\left(B_{R}\right)} .
$$

Denote $E:=\left\{x \in B_{R}: u(x)=0\right\}$ and $u_{\Omega}:={\underset{\Omega}{f}}_{f} u d x$. Then we have

$$
\begin{aligned}
\|u\|_{L_{p}\left(B_{R}\right)}= & \left\|u-u_{E}\right\|_{L_{p}\left(B_{R}\right)} \leq\left\|u-u_{B_{R}}\right\|_{L_{p}\left(B_{R}\right)}+\left|B_{R}\right|^{1 / p}\left|u_{E}-u_{B_{R}}\right| \leq \\
& \leq\left\|u-u_{B_{R}}\right\|_{L_{p}\left(B_{R}\right)}+\frac{\left|B_{R}\right|^{1 / p}}{|E|}\left\|u-u_{B_{R}}\right\|_{L_{1}\left(B_{R}\right)} .
\end{aligned}
$$

Now the result follows from the usual Poincare and Hölder inequalities:

$$
\begin{gathered}
\left\|u-u_{B_{R}}\right\|_{L_{p}\left(B_{R}\right)} \leq c R\|\nabla u\|_{L_{p}\left(B_{R}\right)} \\
\left\|u-u_{B_{R}}\right\|_{L_{1}\left(B_{R}\right)} \leq\left|B_{R}\right|^{1 / p^{\prime}}\left\|u-u_{B_{R}}\right\|_{L_{p}\left(B_{R}\right)} \leq c\left|B_{R}\right|^{1 / n+1 / p^{\prime}}\|\nabla u\|_{L_{p}\left(B_{R}\right)} .
\end{gathered}
$$




\section{References}

[1] J. Bergh, J. Löfström, Interpolation spaces. An introduction. Springer, 1976.

[2] Ch.-Ch. Chen, R.M. Strain, T.-P. Tsai, H.-T. Yau, Lower bounds on the blow-up rate of the axisymmetric Navier-Stokes equations, International Mathematics Research Notices (2008), no. 9.

[3] Ch.-Ch. Chen, R.M. Strain, T.-P. Tsai, H.-T. Yau, Lower bounds on the blow-up rate of the axisymmetric Navier-Stokes equations II, Communications in Partial Differential Equations, 34 (2009), no. 3, 203-232.

[4] L.C. Evans, Partial Differential Equations, Second Edition, AMS, 2010.

[5] N. Filonov, On the regularity of solutions to the equation $-\Delta u+b \cdot \nabla u=0$, Zap. Nauchn. Sem. of Steklov Inst. 410 (2013), 168-186; reprinted in J. Math. Sci. (N.Y.) 195 (2013), no. 1, 98-108.

[6] N. Filonov, T. Shilkin, On some properties of weak solutions to elliptic equations with divergence-free drifts. Mathematical analysis in fluid mechanics: selected recent results, Contemp. Math., 710, Amer. Math. Soc., Providence, RI (2018), 105-120.

[7] S. Friedlander, V. Vicol, Global well-posedness for an advection-diffusion equation arising in magneto-geostrophic dynamics, Ann. Inst. H. Poincare Anal. Non Lineaire, 28 (2011), no. 2, 283-301.

[8] L. Grafakos, Classical Fourier analysis, Springer, New York, 2009.

[9] M. Giaquinta, Multiple Integrals in the Calculus of Variations and Nonlinear Elliptic Systems. Princeton University Press, 1983.

[10] V. Girault, P.-A. Raviart, Finite element approximation of the NavierStokes equations, Springer, 1979.

[11] M. Ignatova, I. Kukavica, L. Ryzhik, The Harnack inequality for secondorder elliptic equations with divergence-free drifts, Comm. Math. Sci., 12 (2014), 681-694.

[12] B. KAnG, H. KIM, $W^{1, p}$-estimates for elliptic equations with lower order terms. Commun. Pure Appl. Anal. 16 (2017), 799-821.

[13] H. Kim, Y. KIM, On weak solutions of elliptic equations with singular drifts. SIAM J. Math. Anal. 47 (2015), no. 2, 1271-1290.

[14] H. Kim, T.-P. Tsai, Existence, uniqueness, and regularity results for elliptic equations with drift terms in critical weak spaces, SIAM J. Math. Anal. 52 (2020), no. 2, 1146-1191.

[15] G. Koch, N. Nadirashvili, G. Seregin, V. Sverak, Liouville theorems for the Navier-Stokes equations and applications. Acta Math. 203 (2009), no. 1, 83-105.

[16] H. Kozono, M. Yamazaki, Uniqueness criterion of weak solutions to the stationary Navier-Stokes equations in exterior domains. Nonlinear Analysis 38 (1999), no. 8, 959-970.

[17] O.A. Ladyzhenskaya, N.N. URaltseva, Linear and quasilinear equations of elliptic type, Academic Press, 1968. 
[18] V. Liskevich, Q.S. Zhang, Extra regularity for parabolic equations with drift terms, Manuscripta Math. 113 (2004), no. 2, 191-209.

[19] V.G. MAZ'JA, I.E. VerbitskiY, Form boundedness of the general secondorder differential operator, Comm. Pure Appl. Math. 59 (2006), 1286-1329.

[20] G. Moscariello, Existence and uniqueness for elliptic equations with lowerorder terms. Adv. Calc. Var. 4 (2011), 421-444.

[21] A.I. Nazarov, N.N. URAltseva, The Harnack inequality and related properties of solutions of elliptic and parabolic equations with divergence-free lowerorder coefficients, St. Petersburg Math. J. 23 (2012), no. 1, 93-115.

[22] R. O'NeIL, Convolution operators and L (p,q) spaces, Duke Math. J. 30 (1963) 129-142.

[23] G. Seregin, Lecture notes on regularity theory for the Navier-Stokes equations. World Scientific Publishing Co. Pte. Ltd., Hackensack, NJ, 2015.

[24] G. Seregin, T. Shilkin, Liouville-type theorems for the Navier-Stokes equations, Russian Math. Surveys 73:4 (2018), 661-724.

[25] G. Seregin, L. Silvestre, V. Sverak, A. Zlatos, On divergence-free drifts. J. Differential Equations 252 (2012), no. 1, 505-540.

[26] G. Seregin, V. Sverak, On type I singularities of the local axi-symmetric solutions of the Navier-Stokes equations, Comm. Partial Differential Equations 34 (2009), no. 1-3, 171-201.

[27] L. Silvestre, V. Vicol, Hölder continuity for a drift-diffusion equation with pressure, Ann. Inst. H. Poincare Anal. Non Lineaire 29 (2012), no. 4, 637-652.

[28] E. Stein, G. Weiss, Introduction to Fourier Analysis on Euclidean Spaces, Princeton University Press, 1971.

[29] M.D. Surnachev, On the uniqueness of solutions to stationary convectiondiffusion equations with generalized divergence-free drift, Complex Variables and Elliptic Equations 63 (2018), no. 7-8, 1168-1184.

[30] T.-P. TsaI, Lectures on Navier-Stokes equations, Graduate Studies in Mathematics, AMS, 2018.

[31] Q.S. Zhang, A strong regularity result for parabolic equations, Commun. Math. Phys. 244 (2004), no. 2, pp. 245-260.

[32] V.V. Zhikov, Remarks on the uniqueness of the solution of the Dirichlet problem for a second-order elliptic equation with lower order terms, Funct. Anal. Appl. 38 (2004), no. 3, 173-183. 